\title{
Appendix A: Supplemental Tables
}

Table A1. Single-Level HCUP CCS Categories Representing a Mental Health-Related Diagnosis

\begin{tabular}{|c|l|}
\hline $\begin{array}{c}\text { Single-Level CCS } \\
\text { Diagnosis } \\
\text { Categories }\end{array}$ & \multicolumn{1}{|c|}{ Ccs Category Description } \\
\hline 650 & Adjustment disorders \\
\hline 651 & Anxiety disorders \\
\hline 652 & Attention-deficit, conduct and disruptive behavior disorders \\
\hline 653 & Delirium, dementia, and amnestic and other cognitive disorders \\
\hline 656 & Impulse control disorders \\
\hline 657 & Mood disorders \\
\hline 658 & Personality disorders \\
\hline 659 & Schizophrenia and other psychotic disorders \\
\hline 660 & Alcohol-related disorders \\
\hline 661 & Substance-related disorders \\
\hline 662 & Suicide and intentional self-inflicted injury \\
\hline 663 & Screening and history of mental health and substance abuse codes \\
\hline 670 & Miscellaneous mental health disorders \\
\hline
\end{tabular}


Table A2. VA Drug Class Codes Representing a Mental Health-Related Drug Fill

\begin{tabular}{|l|l|}
\hline VA Drug Class & \multicolumn{1}{|c|}{ Drug Classification } \\
\hline AD100 & Alcohol Deterrents \\
\hline AD400 & Antidotes, Deterrents, and Poison Control Exchange Resins \\
\hline AD900 & Antidotes/Deterrents, Other \\
\hline CN300 & Sedatives/Hypnotics \\
\hline CN301 & Barbituric Acid Derivative Sedatives/Hypnotics \\
\hline CN309 & Sedatives/Hypnotics, Other \\
\hline CN400 & Anticonvulsants \\
\hline CN500 & Antiparkinson Agents \\
\hline CN600 & Antidepressants \\
\hline CN601 & Tricyclic Antidepressants \\
\hline CN602 & Monamine Oxidase Inhibitor Antidepressants \\
\hline CN609 & Antidepressants, Other \\
\hline CN700 & Antipsychotics \\
\hline CN701 & Phenothiazine/Related Antipsychotics \\
\hline CN709 & Antipsychotics, Other \\
\hline CN750 & Lithium Salts \\
\hline CN800 & CNS Stimulants \\
\hline CN801 & Amphetamines \\
\hline CN802 & Amphetamine Like Stimulants \\
\hline & \\
\hline
\end{tabular}


Table A3. VHA Stop Codes Representing Outpatient Visits

\begin{tabular}{|c|c|l|}
\hline Primary Stop Code & $\begin{array}{c}\text { Secondary } \\
\text { Stop Code }\end{array}$ & \multicolumn{1}{|c|}{ Visit Description } \\
\hline 131 & - & Urgent Care Visit \\
\hline 130 & - & Emergency Room Visit \\
\hline 323,348 & - & Primary Care Visit \\
\hline $156-157,170-178$ & - & Home-Based Primary Care Visit \\
\hline 118,121 & - & Home Health Visit \\
\hline $\begin{array}{c}502,509-510,512,550,557- \\
558,576-577\end{array}$ & 125 & Mental Health Social Work Visit \\
\hline 125 & - & Non-Mental Health Social Work Visit \\
\hline $\begin{array}{c}\text { Any Stop Code not equal to } \\
502,509-510,512,550,557- \\
558,576-577\end{array}$ & 125 & Non-Mental Health Social Work Visit \\
\hline
\end{tabular}

